\title{
The Efficiency Estimation Method of Joint Search and Detection of Objects for Surveillance Technical Systems
}

\author{
Hennadii Khudov ${ }^{1}$, Irina Khizhnyak ${ }^{2}$, Volodymyr Koval ${ }^{3}$, Volodymyr Maliuhaa ${ }^{4}$, Andrii Zvonko ${ }^{5}$, \\ Valerii Yunda $^{6}$, Viacheslav Nagachevskyi ${ }^{7}$, Volodymyr Berezanskyi ${ }^{8}$ \\ ${ }^{1}$ Department of Radar Troops Tactic, Ivan Kozhedub Kharkiv National Air Force University, Kharkiv, Ukraine, \\ 2345kh_hg@ukr.net \\ ${ }^{2}$ Department of Mathematical and Software Automated Control Systems, Ivan Kozhedub Kharkiv National Air \\ Force University, Kharkiv, Ukraine, khizh_ia@ukr.net \\ ${ }^{3}$ Department of Science, Ministry of Defense of Ukraine, Kiev, Ukraine, koval_vol@ukr.net \\ ${ }^{4}$ Department of Anti-aircraft Missiles Tactic, Ivan Kozhedub Kharkiv National Air Force University, Kharkiv, \\ Ukraine, maluga_v@i.ua \\ ${ }^{5}$ Department of Rocket Artillery Armament, Hetman Petro Sahaidachnyi National Army Academy, Lviv, \\ Ukraine, zvonko2008@ukr.net \\ ${ }^{6}$ Department of Rocket Artillery Armament, Hetman Petro Sahaidachnyi National Army Academy, Lviv, \\ Ukraine, valera.yunda@ukr.net \\ ${ }^{7}$ Department of Engineering Equipment, Hetman Petro Sahaidachnyi National Army Academy, Lviv, Ukraine, \\ slavik.inag@gmail.com \\ ${ }^{8}$ Department of Aviation Armament Complexes, Ivan Kozhedub Kharkiv National Air Force University, Kharkiv, \\ Ukraine, berwog75@gmail.com
}

\begin{abstract}
The article proposes the efficiency estimation method of joint search and detection of objects for surveillance technical systems. The differential characteristics of the Bayes criterion of minimum average risk, a priori probabilities of hypotheses about the absence of an object and its presence are taken into account in the calculations. An algorithm has been developed for calculating the unconditional probability of detecting an object of observation during a joint search and detection of objects in technical surveillance systems. Shown, that a joint search and detection of the objects of surveillance using a uniformly optimal search strategy provides a higher unconditional probability of the correct detection of the object of surveillance.
\end{abstract}

Key words : surveillance technical system, the object of surveillance, area of view, joint search and detection of objects, the unconditional probability of correct detection, the search potential.

\section{INTRODUCTION}

Issues of search and detection of objects of surveillance in conditions of rapid development information technologies are of significant interest [1-5].

Today, technical surveillance systems solve the problem of searching and detecting objects of surveillance in conditions of limited search potential [1-3, 6-9]. This is relevant for both radar [6-8, 10-13] and optical [14-20] surveillance systems.

Previously, a method for joint optimization of continuous and discrete search and detection of objects in information systems was developed $[1,3]$. The Bayes optimal rules for decision making for joint optimizing of continuous and discrete search and detection of objects are formulated [2, 21-22]. But evaluating the effectiveness of collaborative search and object discovery was not considered.

\subsection{Problem analysis}

In the classic theory of detection the main indexes of the effectiveness of detection are [5, 21-22]:

$P\left(\gamma_{1} / H_{0}, \mathbf{X}\right)-$ the conditional probability of false alarm;

$P\left(\gamma_{1} / H_{1}, \mathbf{X}\right)$ - the conditional probability of correct detection;

where $H_{0}$ - hypothesis about the absence of an object in the viewing area;

$H_{1}$ - hypothesis about the presence of an object in the viewing area;

$\mathbf{X}\left(x_{1}, \mathbf{x}_{2}\right)$ - object's coordinate. 
The conditional probability of false alarm and correct detection is calculated taking into account the statistical characteristics of the signals that are received from objects of surveillance.

These indicators $\left(P\left(\gamma_{1} / H_{0}, \mathbf{X}\right), P\left(\gamma_{1} / H_{1}, \mathbf{X}\right)\right)$ take into account only the detection characteristics of objects and do not take into account the effectiveness of the search for objects.

In [1] for joint search and detection of objects the differential characteristics of the Bayes criterion of minimum average risk are used. These characteristics allow us to move on to assessing the effectiveness of joint search and detection of objects.

In [1-3], the effectiveness of the joint search and detection of objects of surveillance is estimated by the value of the following performance indexes, namely:

$P_{0}\left(\gamma_{1}, t\right)$ - the unconditional probability of false alarm;

$P_{1}\left(\gamma_{1}, t\right)$ - the unconditional probability of correct detection;

average time to detect the object of surveillance, where $\gamma_{1}$ is the decision made that the object of surveillance was detected;

$t$ - the time.

These indicators $\left(P_{0}\left(\gamma_{1}, t\right), P_{1}\left(\gamma_{1}, t\right)\right)$ take into the differential characteristics of the Bayes criterion of minimum average risk.

As additional indexes of the effectiveness of joint search and detection, it is advisable to save indexes that are developed in the classic theory of detection $\left(P\left(\gamma_{1} / H_{0}, \mathbf{X}\right)\right.$, $\left.P\left(\gamma_{1} / H_{1}, \mathbf{X}\right)\right)$.

Let us consider an algorithm for calculating the unconditional probability of the correct detection of an object of surveillance and the average time for detection of this object.

\section{MAIn MATERIAL}

We will calculate the unconditional probability of the correct identification of the object and the average time to detect the object in a joint search and detection in two coordinates for a particular case. Namely, when the a priori probability density of the location of the object is set in the form of a truncated normal law, and the viewing area is a circle with a radius of $S$.

We assume that the amplitude of the signal that is received is distributed according to the Nakagami distribution.
Define function $\lambda(x, t)$ of search strategy

$$
\lambda_{1}\left(x_{1}, x_{2} ; t\right)=\left\{\begin{array}{c}
L_{0} / z(t) ; \text { for } x_{1}^{2}+x_{2}^{2}<r^{2}(t) ;(1) \\
0 ; \text { for } x_{1}^{2}+x_{2}^{2} \geq r^{2}(t),
\end{array}\right.
$$

where $z(t)$ - a measure of the current search area;

$L_{0}$ - the search potential of a surveillance technical system.

This search strategy function will be distributed in a circle centered in the center of the viewing area and the current radius $r(t)$.

This will happen until the scope of the search strategy becomes equal to the size of the viewing area.

We define this moment of time $t_{1}$ on condition that the dimensions of the current viewing area are equal to the dimensions of the entire area of view. We get the expression (2):

$$
\sigma \sqrt{2 \pi L_{0} t}=\pi S^{2}
$$

where $\sigma$ - standard deviation in the a priori law of the location of the object. from expression (2) we obtain:

$$
t_{1}=\frac{\pi S^{4}}{4 \sigma^{2} L_{0}} \text {. }
$$

In the points of the viewing area where the search strategy is distributed, the search potential will be accumulated over time $\left[0, t_{1}\right]$. This search potential is proportional to the detection parameter, which can be calculated according to expression (4):

$$
\varphi_{1}=\int_{t\left(x_{1}, x_{2}\right)}^{t_{1}} \lambda\left(x_{1}, x_{2}\right) d x
$$

where $t\left(x_{1}, x_{2}\right)$ - function that matters the start time of viewing the points of the viewing area.

We find this function $t\left(x_{1}, x_{2}\right)$ under condition (5):

$$
x_{1}^{2}+x_{2}^{2}=2 \sigma \sqrt{\frac{L_{0} t\left(x_{1}, x_{2}\right)}{\pi}},
$$

From (5) we obtain the expression (6):

$$
t\left(x_{1}, x_{2}\right)=\frac{\pi\left(x_{1}^{2}+x_{2}^{2}\right)^{2}}{4 \sigma^{2} L_{0}} .
$$


We substitute (1), (3) and (6) into (4). Take the integral (4) and get the expression (7):

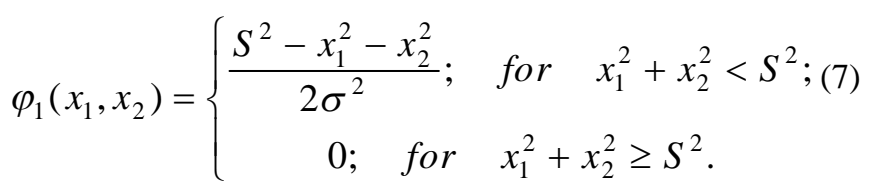

To find function $\varphi_{1}\left(x_{1}, x_{2}\right)$ at any moment of time, we replace in (4) the upper integration boundary for the current time $t$.

As a result we get the expression (8):

$$
\varphi_{1}\left(x_{1}, x_{2}\right)=\int_{t\left(x_{1}, x_{2}\right)}^{t} \lambda_{1}\left(x_{1}, x_{2} ; t\right) d t
$$

After time moment $t_{1}$ at the accepted density of a priori probability, the search strategy will be distributed under conditions of uniform distribution density (base).

While the current viewing area $\Omega(t)$ will completely coincide with the entire viewing area, and

$$
\Omega(t)=\pi S^{2} .
$$

In this case, taking into account expression (9), the search strategy has the following form:

$$
\lambda_{2}\left(x_{1}, x_{2} ; t\right)=\left\{\begin{array}{ccc}
\frac{L_{0}}{\pi S^{2}} ; & \text { for } & x_{1}^{2}+x_{2}^{2}<S^{2} \\
0 ; & \text { for } & x_{1}^{2}+x_{2}^{2} \geq S^{2}
\end{array}\right.
$$

In time interval $\left[t_{1}, T\right]$, the search potential, taking into account expression (10), can be determined according to expression (11):

$$
\varphi_{2}\left(x_{1}, x_{2}\right)=\int_{t_{1}}^{T} \lambda_{2}\left(x_{1}, x_{2} ; t\right) d t
$$

We substitute (10), (3) into (11). As a result we get the expression (12):

$$
\varphi_{2}\left(x_{1}, x_{2}\right)=\frac{L_{0}\left(T-t_{1}\right)}{\pi S^{2}},
$$

where $T$ - time to view the entire viewing area.

We calculate the unconditional probability of the correct detection of the object during the search $T$ when continuously searching for objects in two coordinates in a circular viewing area.
In this case, we will take into account the proportional dependence of the detection parameter and the calculated value of the search potential. We restrict ourselves to the case of detecting a signal with an amplitude that is distributed according to the Nakagami distribution with coefficient $m$.

If $m=1$, the Nakagami distribution is converted into a Rayleigh distribution.

If $m=2$, the Nakagami distribution is converted into a Swerling distribution.

For the convenience of subsequent calculations, we introduce a polar coordinate system. Then, taking into account the transition to the polar coordinate system and the transition in both parts of the expression to dimensionless quantities by multiplying by the search time, expressions (1) and (10) will have the form (13):

$$
\lambda\left(\rho_{1}, \beta, s\right) T=\left\{\begin{array}{cc}
\frac{k^{1 / 2}}{2(\pi s)^{1 / 2}} ; \quad \rho_{1}<h ; 0 \leq \beta \leq 360^{\circ} \\
\frac{k}{\pi h^{2}} ; \quad \rho_{1}<h ; 0 \leq \beta \leq 360^{\circ}
\end{array}\right.
$$

where $k$ - value that determines the total search potential of the surveillance technical system;

$h$ - value that determines the size of the entire viewing area.

And for the search potential when changing the value of $s$ in the interval of expression (7), expression (8) is converted to the next expression (14):

$$
\begin{gathered}
\varphi\left(\rho_{1}, \beta\right)=\frac{h^{2}}{4}+\frac{k^{2}}{\pi h^{2}}-\frac{\rho_{1}^{2}}{2} \\
\rho_{1}<h ; \quad 0 \leq \beta \leq 360^{0} .
\end{gathered}
$$

Thus, the expressions for the unconditional probability of the correct detection of the object of surveillance:

for $m=1$

$$
\begin{aligned}
& P_{1}\left(\gamma_{1}, T\right)=\frac{1}{1-e^{-\frac{h^{2}}{2}} \times} \\
& \times \int_{0}^{h} \exp \left(-\frac{\ln (1 / F)}{1+\frac{h^{2}}{4}+\frac{k^{2}}{\pi h^{2}}-\frac{\rho_{1}^{2}}{2}}-\frac{\rho_{1}^{2}}{2}\right) \rho_{1} d \rho
\end{aligned}
$$

for $m=2$ 


$$
\begin{aligned}
& P_{1}\left(\gamma_{1}, T\right)=\frac{1}{1-e^{-\frac{h^{2}}{2}} \times} \\
& \quad\left(\left(1+\frac{\frac{h^{2}}{8}+\frac{k^{2}}{2 \pi h^{2}}-\frac{\rho_{1}^{2}}{4}}{\left(1+\frac{h^{2}}{8}+\frac{k^{2}}{2 \pi h^{2}}-\frac{\rho_{1}^{2}}{4}\right)^{2}} \ln \left(\frac{1}{F}\right)\right) \times\right. \\
& \times \int_{0}^{h} \quad \rho_{1} d \rho ; \\
& \quad \times \exp \left(-\frac{\ln \left(\frac{1}{F}\right)}{1+\frac{h^{2}}{8}+\frac{k^{2}}{2 \pi h^{2}}-\frac{\rho_{1}^{2}}{4}}-\frac{\rho_{1}^{2}}{2}\right)
\end{aligned}
$$

We calculate the unconditional probability of correct detection according to expressions (15) and (16). Figures 1 and 2 show the dependences of the calculated values on a value that characterizes the speed and time of viewing the entire viewing area.

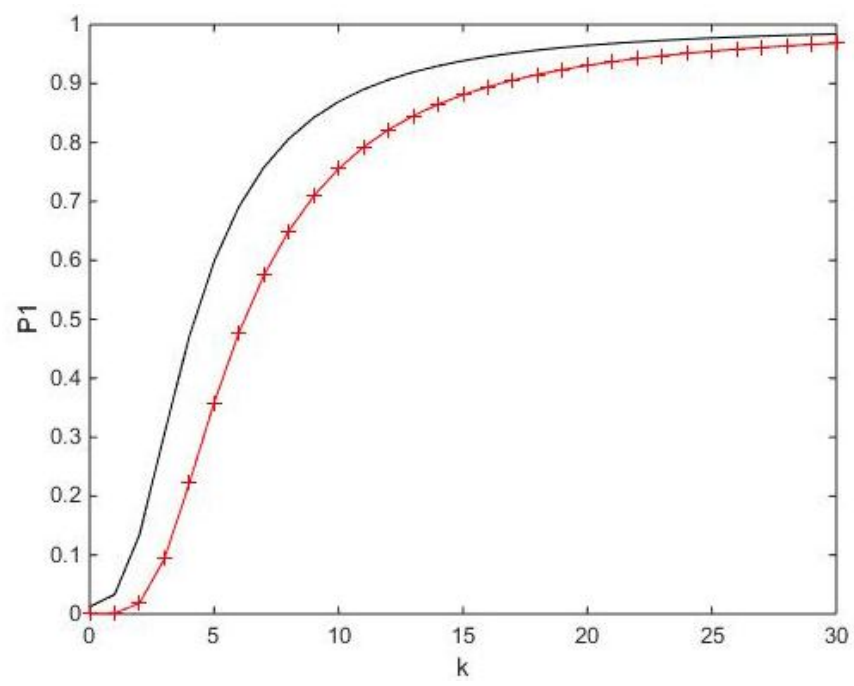

Figure 1: The dependence of the unconditional probability of correct detection on the value of $k$ at $F=10^{-2}$ (upper curve),

$$
F=10^{-4} \text { (lower curve) }(m=1, h=1)
$$

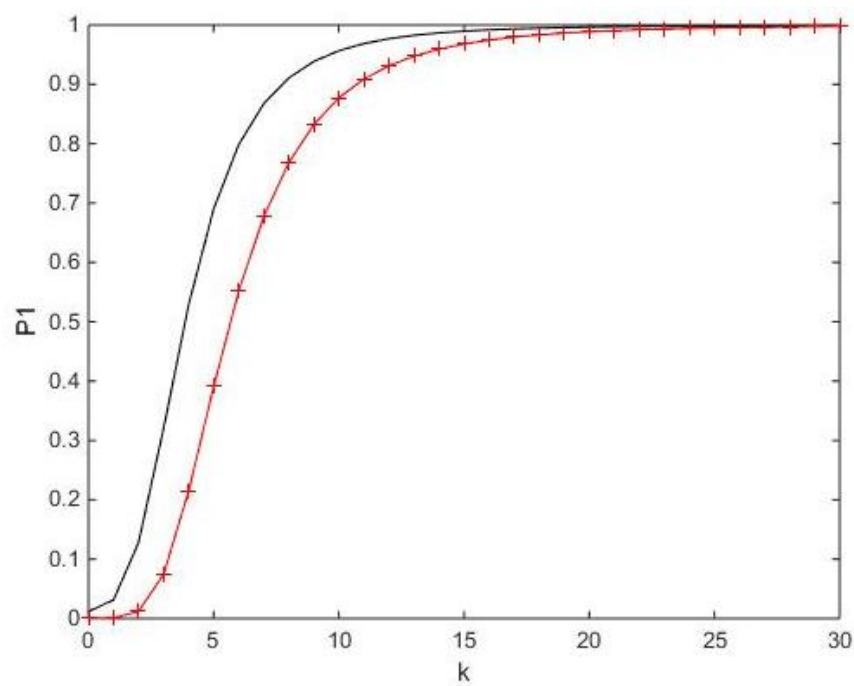

Figure 2: The dependence of the unconditional probability of correct detection on the value of $k$ at $F=10^{-2}$ (upper curve),

$$
F=10^{-4} \text { (lower curve) }(m=2, h=1)
$$

Figures 1 and 2 show the dependence of the unconditional probability of correct detection on a value of $k$ for the values of the conditional probability of false alarm $F=10^{-2}$ (upper curve) and $F=10^{-4}$ (lower curve).

The graphs shown in Figures 1 and 2 were calculated under the following conditions:

Fig. 1: $m=1, h=1$;

Fig. 2: $m=2, h=1$.

The minimum value of the value, which is proportional to the value of the total search potential of the surveillance system, is selected subject to mandatory viewing of the entire viewing area.

So from this statement we get condition (17):

$$
k \geq \frac{\pi h^{4}}{4}
$$

To assess the effectiveness of the search algorithm and object detection using a uniformly optimal search strategy, we will compare this algorithm with the known algorithms of the space-temporal distribution of search efforts in surveillance systems.

Provided that the known algorithms assume a uniform distribution of the search potential across the viewing area. With a uniform distribution, the expression for the detection parameter, and, consequently, for the search potential, has the following form:

$$
\varphi\left(x_{1}, x_{2}\right)=\frac{L_{0} T}{\pi S^{2}}
$$

which, taking into account the introduced notation, has the form:

$$
\varphi\left(x_{1}, x_{2}\right)=\frac{k}{\pi h^{2}} .
$$

The expressions for the conditional probability of the correct surveillance of the object under surveillance with a uniform 
Hennadii Khudov et al., International Journal of Emerging Trends in Engineering Research, 8(3), March 2020, 813 - 819

distribution of the search potential over the search area, taking into account expression (19), have the following form:

for $m=1$

$$
P_{1}\left(\gamma_{1} / H_{1}, x_{1}, x_{2}\right)=F^{\frac{1}{1+\frac{k}{\pi h^{2}}}},
$$

for $m=2$

$$
\begin{aligned}
& P_{1}\left(\gamma_{1} / H_{1}, x_{1}, x_{2}\right)=\left(1+\frac{k}{2 \pi h^{2}\left(1+\frac{k}{\pi h^{2}}\right)^{2}} \times\right. \\
& \left.\times \ln \left(\frac{1}{F}\right)\right) F^{\frac{1}{1+\frac{k}{\pi h^{2}}}} .
\end{aligned}
$$

Using expressions (20) and (21) we get the unconditional probability of the correct detection of the object with a uniform distribution of the search potential across the viewing area. We will use the notation introduced earlier:

for $m=1$

$$
P_{1}\left(\gamma_{1}, T\right)=\frac{F^{\frac{1}{1+\frac{k}{\pi h^{2}}}}}{1-e^{-\frac{h^{2}}{2}}} \int_{0}^{h} \exp \left(-\frac{\rho_{1}^{2}}{2}\right) \rho_{1} d \rho_{1},
$$

for $m=2$

$$
\begin{aligned}
& P_{1}\left(\gamma_{1}, T\right)=\frac{F^{\frac{1}{1+\frac{k}{\pi h^{2}}}}\left(1+\frac{k \ln \left(\frac{1}{F}\right)}{2 \pi h^{2}\left(1+\frac{k}{2 \pi h^{2}}\right)^{2}}\right)}{1-e^{-\frac{h^{2}}{2}}} \times \\
& \times \int_{0}^{h} \exp \left(-\frac{\rho_{1}^{2}}{2}\right) \rho_{1} d \rho_{1} .
\end{aligned}
$$

Figures 3 and 4 show graphs of the dependence of the unconditional probability of detecting an object:

the lower curve - when a uniform distribution of the search potential across the viewing area, which are calculated by the expressions (22) and (23) versus the value of $k$;

the upper curve - when searching and discovering objects together, which are calculated by the expressions (15) and (16) versus value of $k$.
While the graphs that are shown in Fig. 3 and 4 are calculated under the following conditions:

Fig. 3: $m=1, h=1$;

Fig. 4: $m=2, h=1$.

The value of the conditional probability of false alarm was taken equal to $F=10^{-3}$.

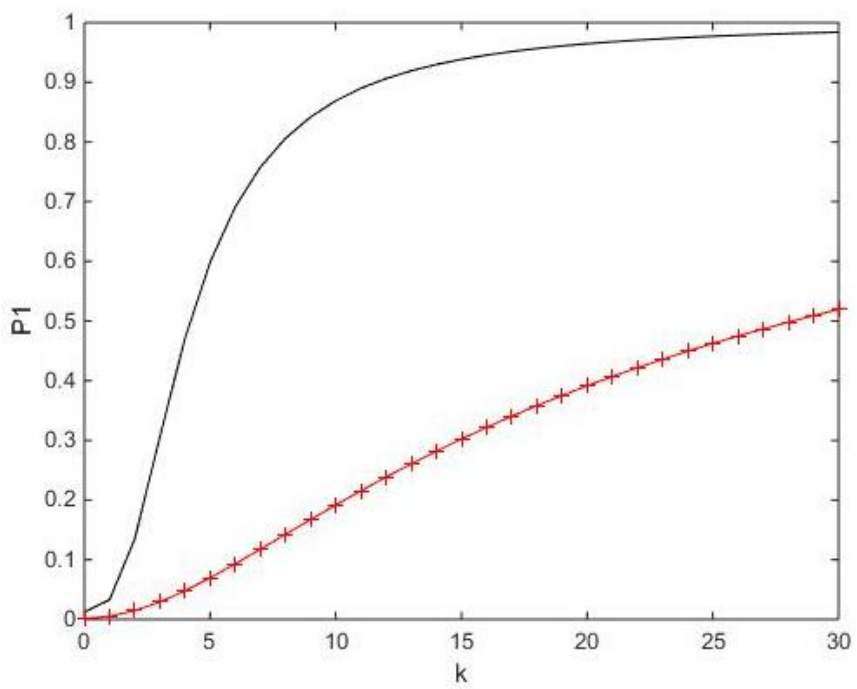

Figure 3: The dependence of the unconditional probability of correct detection on the value of $k$ for a uniform distribution of the search potential (lower curve) and in the joint search and detection of the object (upper curve) $(m=1, h=1)$

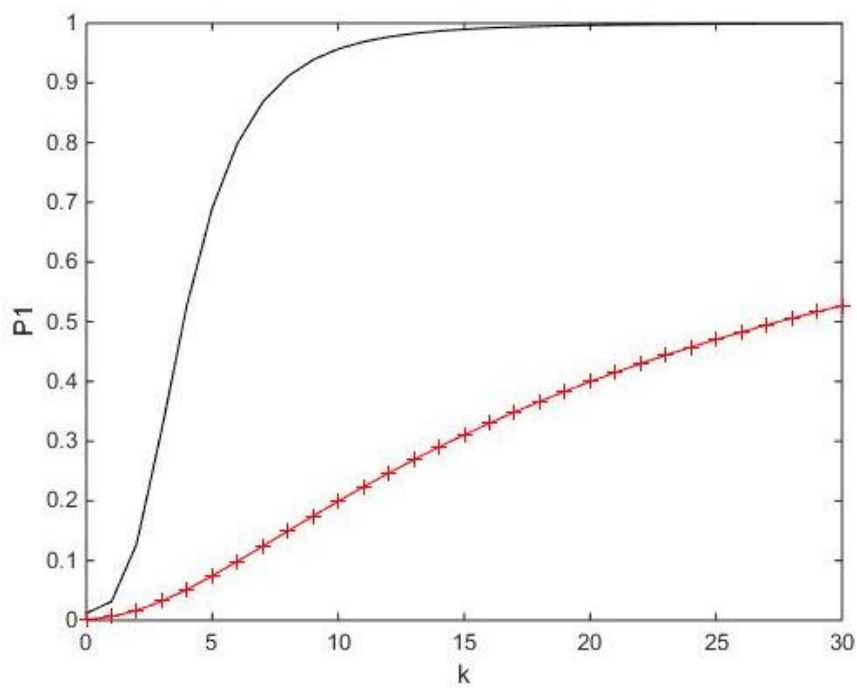

Figure 4: The dependence of the unconditional probability of correct detection on the value of $k$ for a uniform distribution of the search potential (lower curve) and in the joint search and detection of the object (upper curve) $(m=2, h=1)$

From these graphs it can be concluded that a joint search and detection of the object of surveillance using a uniformly optimal search strategy provides a higher unconditional 
probability of the correct detection of the object of surveillance. This is especially noticeable provided that there is a strict restriction on the value of $k$.

\section{CONCLUSION}

Thus, the article proposes the efficiency estimation method of joint search and detection of objects for surveillance technical systems. The differential characteristics of the Bayes criterion of minimum average risk, a priori probabilities of hypotheses about the absence of an object and its presence are taken into account in the calculations.

An algorithm has been developed for calculating the unconditional probability of detecting an object of observation during a joint search and detection of objects in technical surveillance systems.

Shown, that a joint search and detection of the objects of surveillance using a uniformly optimal search strategy provides a higher unconditional probability of the correct detection of the object of surveillance.

In future research, it is necessary to assess the average time that is needed to detect the object of surveillance during the joint search and detection of objects and uniform distribution of the search potential of technical surveillance systems.

\section{REFERENCES}

1. H. Khudov, I. Khizhnyak, F. Zots, G. Misiyuk, and O. Serdiuk. The Bayes Rule of Decision Making in Joint Optimization of Search and Detection of Objects in Technical Systems, IJETER, № 8(1), 2020, pp. 7-12. DOI: https://doi.org/10.30534/ijeter/2020/02812020.

2. H. Khudov, A. Zvonko, I. Khizhnyak, V. Shulezko, V. Khlopiachyi, V. Chepurnyi, and I. Yuzova. The Synthesis of the Optimal Decision Rule for Detecting an Object in a Joint Search and Detection of Objects by the Criterion of Maximum Likelihood, IJETER, № $8(2), \quad 2020, \quad$ pp. 520-524. DOI: https://doi.org/10.30534/ijeter/2020/40822020.

3. H. Khudov, I. Khizhnyak, I. Yuzova, O. Baranik, G. Semiv, S. Bondarenko, and O. Tytarenko. The Optimization Technique for Joint Discrete Search and Detection of Observation Objects, IJETER, № 8(2), 2020, $\quad$ pp. 533-538. DOI: https://doi.org/10.30534/ijeter/2020/42822020.

4. H. Khudov, O. Makoveychuk, I. Khizhnyak, I. Yuzova , A. Irkha, and V. Khudov. The Mosaic Sustainable Marker Model for Augmented Reality Systems, IJATCSE, № 9(1), 2020, pp. 637-642. DOI: https://doi.org/10.30534/ijatcse/2020/89912020.

5. K. Indira, and U. Sakthi. A Comparative Analysis on Intrusion Detection System for SDWSN using Ensemble Classifier, IJETER, № 8(2), 2020, pp. 551-556.

DOI: https://doi.org/10.30534/ijeter/2020/45822020.
6. H. Khudov, A. Zvonko, S. Kovalevskyi, V. Lishchenko, and F. Zots. Method for the detection of smallsized air objects by observational radars, Eastern-European Journal of Enterprise Technologies, № 2/9 (92), 2018, pp. 61-68.

DOI:https://doi.org/10.15587/1729-4061.2018.126509.

7. V. Atamanyuk, A. Zvonko, Y. Kosovtsov, and Y. Shabatura. Nonuniform absorbing coating as an effective way to reduce radar visibility of an object with the surface formed by flat conductive plates, 2017 IEEE First Ukraine Conference on Electrical and Computer Engineering (UKRCON), 2017, pp. 75-79.

8. V. Lishchenko, V. Chaliy, H. Khudov, and A. Zvonko. Proposals for Improving of Air Surveillance Informativity in MIMO Radar Systems Based on Two-Dimensional Radars, in Intern. Scient.-Pract. Conf. Problems of Infocommunications. Science and Technology (PIC S\&T), 2018, pp. 153-156. DOI: https://doi.org/10.1109/infocommst.2018.8632052.

9. O. Lemeshko, H. Kozlova, and A. Romanyuk. Flow-based model of fault-tolerant routing in MPLS-network, in Intern. Crimean Conference Microwave and Telecommunication Technology, Conference Proceedings (CriMiCo), 2013.

10. H. Khudov, A. Fedorov, D. Holovniak, and G. Misiyuk. Improving the Efficiency of Radar Control of Airspace with the Multilateration System Use, in Intern. Scient.-Pract. Conf. Problems of Infocommunications. Science and Technology (PIC S\&T), 2018, pp. 680-684. DOI: https://doi.org/10.1109/infocommst.2018.8632141.

11. V. Lishchenko, H. Khudov, V. Tiutiunnyk, V._Kuprii, F.ZZots, and G._Misiyuk. The Method of Increasing the Detection Range of Unmanned Aerial Vehicles In Multiradar Systems Based on Surveillance Radars, in 2019 IEEE 39th International Conference on Electronics and Nanotechnology (ELNANO), 2019. DOI: https://doi.org/10.1109/ELNANO.2019.8783263.

12. M. Iasechko, M. Mozhaiev, I. Manzhai, M. Volk, V. Manoylo, O. Ochkurenko, D. Maksuita, V. Larin, A. Markov, and O. Kostyria. Conditions for Reliable Transmission of Information Over Long Distances Using a Powerful Electromagnetic Radiation, IJATCSE. $\quad 8(1), \quad 2020, \quad$ pp. 138-144. DOI: https://doi.org/10.30534/ijeter/2020/18812020.

13. M. Iasechko, Y. Gnusov, I. Manzhai, O. Uhrovetskyi, V. Manoylo, A. Iesipov, O. Zaitsev, M. Volk, and O. Volk. Determination of Requirements for the Protection of Radio-Electronic Equipment from the Terroristic Influence by Electromagnetic Radiation, IJATCSE. 7(12), 2019, pp. 772-777. DOI: https://doi.org/10.30534/ijeter/2019/077122019.

14. I. Ruban, H. Khudov, O. Makoveichuk, I. Khizhnyak, V. Khudov, V. Podlipaiev, V. Shumeiko, O. Atrasevych, A. Nikitin, and R. Khudov. Segmentation of opticalelectronic images from on-board systems of remote sensing of the Earth by the artificial bee colony method, Eastern-European Journal of Enterprise 
Technologies, № 2/9 (98), 2019, pp. 37-45. DOI: https://doi.org/10.15587/1729-4061.2019.161860.

15. H. Khudov, I. Ruban, O. Makoveichuk, H. Pevtsov, V. Khudov, I. Khizhnyak, S. Fryz, V. Podlipaiev, Y. Polonskyi, and R. Khudov. Development of methods for determining the contours of objects for a complex structured color image based on the ant colony optimization algorithm, Eureka: Physics and Engineering, № 1, 2020, pp. 34-47. DOI: https://doi.org/10.21303/2461-4262.2020.001108.

16. I. Ruban, V. Khudov, O. Makoveichuk, H. Khudov, and I. Khizhnyak. A Swarm Method for Segmentation of Images Obtained from On-Board Optoelectronic Surveillance Systems, in Intern. Scient.-Pract. Conf. Problems of Infocommunications. Science and Technology (PIC S\&T), 2018, pp. 613-618. DOI: https://doi.org/10.1109/infocommst.2018.8632045.

17. I. Ruban, H. Khudov, V. Khudov, I. Khizhnyak, and O. Makoveichuk. Segmentation of the images obtained from onboard optoelectronic surveillance systems by the evolutionary method, Eastern-European Journal of Enterprise Technologies, № 5/9 (89), 2017, pp. 49-57. DOI: https://doi.org/10.15587/1729-4061.2017.109904.

18. I. Ruban, H. Khudov, O. Makoveichuk, M. Chomik, V. Khudov, I. Khizhnyak, V. Podlipaiev, Y. Sheviakov, O. Baranik, and A. Irkha. Construction of methods for determining the contours of objects on tonal aerospace images based on the ant algorithms, Eastern-European Journal of Enterprise Technologies, № 5/9 (101), 2019, pp. 25-34. https://doi.org/10.15587/1729-4061.2019.177817.

19. Ruban I., Khudov H., Makoveichuk O., Khizhnyak I., Lukova-Chuiko N., Pevtsov G., Sheviakov Y., Yuzova I., Drob Y., and Tytarenko O. Method for determining elements of urban infrastructure objects based on the results from air monitoring, Eastern-European Journal of Enterprise Technologies, № 4/9 (100), 2019, pp. 52-61.

DOI: https://doi.org/10.15587/1729-4061.2019.174576.

20. I. Ruban, V. Khudov, H. Khudov, and I. Khizhnyak. An Improved Method for Segmentation of a Multiscale Sequence of Optoelectronic Images, in Intern. Scient.-Pract. Conf. Problems of Infocommunications. Science and Technology (PIC S\&T), 2017, pp. 137-140. DOI: https://doi.org/10.1109/INFOCOMMST.2017.8246 367.

21.J. Li, X. Chu, W. He, F. Ma, R. Malekian, and Z. Li. A Generalised Bayesian Inference Method for Maritime Surveillance Using Historical Data, Symmetry, № 11(2), $188,2019$. DOI: https://doi.org/10.3390/sym11020188.

22. N. Petrović, L. Jovanov, A. Pizurica, and W. Philips. Object Tracking Using Naïve Bayesian Classifiers, Advanced Concepts for Intelligent Vision Systems: $10^{\text {th }}$ International Conference (ACIVS 2008), 2008, pp. 775-784.

DOI: https://doi.org/10.1007/978-3-540-88458-3_70. 